\title{
Pacemaker lead infection: echocardiographic features, management, and outcome
}

\author{
F Victor, C De Place, C Camus, H Le Breton, C Leclercq, D Pavin, P Mabo, C Daubert
}

\begin{abstract}
Objective-To compare transthoracic and transoesophageal echocardiography (TTE, TOE) in patients with permanent pacemaker lead infection and to evaluate the safety of medical extraction in cases of large vegetations.

Methods-TTE and TOE were performed in 23 patients with definite pacemaker lead infection. Seventeen patients without previous infection served as a TOE reference for non-infected leads.
\end{abstract}

Results-TTE was positive in seven cases $(30 \%)$ whereas with TOE three different types of vegetations attached to the leads were visualised in 21 of the 23 cases $(91 \%)$. Of the 20 patients with vegetations and lead culture, 17 (85\%) had bacteriologically active infection. Left sided valvar endocarditis was diagnosed in two patients. In the control group, strands were visualised by TOE in five patients, and vegetations in none. Medical extraction of vegetations $\geqslant 10 \mathrm{~mm}$ was performed in 12 patients and was successful in nine $(75 \%)$ without clinical pulmonary embolism. After 31.2 (19.1) months of follow up (mean (SD)), all patients except one were cured of infection; three died from other causes.

Conclusions-Combined with bacteriological data, vegetations seen on TOE strongly suggest pacemaker lead infection. Normal TTE examinations do not exclude this diagnosis because of its poor sensitivity. Medical extraction of even large vegetations appeared to be safe. (Heart 1999;81:82-87)

Keywords: pacemaker lead infection; transoesophageal echocardiography

Infection secondary to cardiac pacemaker implantation is a major factor in morbidity and mortality. ${ }^{1}$ The incidence of infection following implantation has been variably assessed as ranging from $0.13 \%{ }^{2}$ to $19.9 \%$. $^{3}$ Early diagnosis and accurate localisation of infection along the lead or on the endocardium are two major issues. Local inflammation at the site of the pulse generator pocket clearly indicates infection. On the other hand, sepsis involving only the lead raises major diagnostic problems that may result in delayed treatment and poor outcome. ${ }^{4}$ Optimal management is still controversial, although complete cure of infection requires removal of the entire pacing system in most cases. The safety of medical extraction of large vegetations $(\geqslant 10 \mathrm{~mm})$ remains uncertain.

The aims of this study were: (1) to describe the clinical and microbiological characteristics of patients presenting with pacemaker lead infection; (2) to compare transthoracic echocardiography (TTE) and transoesophageal echocardiography (TOE); (3) to describe the echocardiographic findings; (4) to compare echocardiographic observations with the result of culture of the explanted leads; and (5) to evaluate medical extraction of vegetations of $\geqslant 10 \mathrm{~mm}$.

\section{Methods}

PATIENTS

We studied prospectively 23 patients who were admitted to hospital between 1992 and 1996 and in whom definite pacemaker lead infection was diagnosed. There were 16 men and seven women aged from 32 to 88 years (mean (SD) 65.3 (15.1) years). The diagnosis was based on the presence of at least three of the following four criteria: clinical signs of sepsis, positive blood cultures, visualisation of neostructures consistent with vegetations on echocardiography, ${ }^{6}$ or microbiological documentation of lead infection after removal regardless of TOE findings.

We documented the clinical characteristics of the patients, the portal of entry, the delay after the last pacemaker procedure, the duration of symptoms before diagnosis, and the microbiological data. The explantation technique, reimplantation, and outcome were evaluated.

The time interval between the last intervention and the onset of infection distinguished early (up to two weeks), intermediate (two weeks to six months), and late onset infections (more than six months). ${ }^{7}$

ECHOCARDIOGRAPHIC STUDY

Each patient underwent TTE and TOE (Sonos 1000; Hewlett Packard, Boston, USA) with 2.5 and $5 \mathrm{MHz}$ single plane probes initially, then with a multiplane probe for the final 10 patients. TOE (multiplane) was also performed in 17 control pacemaker patients without any sign of infection following pacemaker implantation. This group enabled us to define an echocardiographic reference for a normal intracardiac pacemaker lead. TOE was performed with the patients awake and fasted for at least four hours. Premedication consisted of lignocaine spray and intravenous sedation with midazolam. The examinations were recorded on videotape for subsequent analysis by three different operators, one of whom had no
Accepted for publication 18 June 1998 
Table 1 Clinical characteristics of the 23 patients with pacemaker lead infection

\begin{tabular}{|c|c|c|c|c|c|c|c|}
\hline Case & Age/sex & Indication & Pacing system & $\begin{array}{l}\text { Number } \\
\text { of leads }\end{array}$ & $\begin{array}{l}\text { Reoperation(s) } \\
\text { before infection }\end{array}$ & $\begin{array}{l}\text { Time from the last } \\
\text { operation to infection } \\
\text { (months) }\end{array}$ & Portal of entry \\
\hline 1 & $38 / \mathrm{M}$ & BTS/ARVC & VVI & 2 & GR, ER & 19 & Cutaneous \\
\hline 2 & $50 / \mathrm{M}$ & AVB III & DDD & 2 & No & 11 & Unknown \\
\hline 3 & $88 / \mathrm{M}$ & AVB II & DDD & 2 & No & 13 & Unknown \\
\hline 4 & $32 / \mathrm{M}$ & ARVC & ICD & 2 & PR & 15 & Unknown \\
\hline 5 & $53 / \mathrm{F}$ & AVB III & DDD & 4 & ER & 7 & Unknown \\
\hline 6 & $67 / \mathrm{M}$ & AVB III & DDD & 2 & GR & 5 & Cutaneous \\
\hline 7 & $48 / \mathrm{M}$ & $\mathrm{AF}$ & AAI/VVI & 2 & GR, ER & 2 & Unknown \\
\hline 8 & $69 / \mathrm{F}$ & AVB III & VVIR & 1 & No & 13 & Unknown \\
\hline 9 & $73 / \mathrm{M}$ & AVB III & DDD & 2 & GR & 28 & Unknown \\
\hline 10 & $79 / \mathrm{M}$ & AVB III & VVI & 1 & GR & 3 & Generator \\
\hline 11 & $68 / M$ & AVB III & DDD & 1 & GR, ER & 31 & Unknown \\
\hline 12 & $55 / \mathrm{M}$ & Unknown & VVI/VDI & 2 & GR, ER & 11 & Generator \\
\hline 13 & $75 / \mathrm{M}$ & $\mathrm{V}$ & VVI & 1 & No & 91 & Unknown \\
\hline 14 & $82 / \mathrm{M}$ & AVB III & VVI & 1 & GR, ER & 11 & Generator \\
\hline 15 & $68 / \mathrm{M}$ & AVB III & DDD & 2 & ER & 8 & Generator \\
\hline 16 & $47 / \mathrm{M}$ & AVB III & VVIR & 2 & No & 1 & Generator \\
\hline 17 & $72 / \mathrm{M}$ & BTS & DDD & 2 & No & 0 & Unknown \\
\hline 18 & $73 / \mathrm{M}$ & AVB III & DDD & 3 & $\mathrm{ER}, \mathrm{RF}$ & 45 & Unknown \\
\hline 19 & $79 / \mathrm{F}$ & $\mathrm{V}$ & DDD & 2 & No & 67 & Unknown \\
\hline 20 & $62 / \mathrm{F}$ & V & VVI & 1 & No & 74 & Unknown \\
\hline 21 & $77 / \mathrm{F}$ & BTS & DDD & 2 & No & 0 & Generator \\
\hline 22 & $61 / \mathrm{F}$ & AVB II & DDD & 2 & No & 27 & Unknown \\
\hline 23 & $86 / \mathrm{F}$ & BTS & VVI & 1 & GR & 120 & Digestive \\
\hline
\end{tabular}

AF, atrial fibrillation; ARVC, arrhythmogenic right ventricular cardiomyopathy; AVB, atrioventricular block; BTS, bradycardiatachycardia syndrome; ER, electrode replacement; F, female; GR, generator replacement; M, male; PR, patch replacement; RF, radiofrequency catheter ablation of the AV node; $\mathrm{V}$, vasovagal syncope.

knowledge of the patients' medical records. The controls were examined by one operator, who focused on investigating neostructures along the intracardiac route of the pacemaker leads.

Tricuspid valve endocarditis was defined by the existence of valve leaflet mutilation or vegetation, or both, or annular abscess. Strands were defined as fine, mobile, echodense structures, attached to the leads, $1-2 \mathrm{~mm}$ wide and a few millimetres long. ${ }^{8}$

\section{Results}

CLINICAL CHARACTERISTICS

Clinical characteristics are given in table 1 . Only one patient had a defibrillator implanted, 10 had a single chamber pacemaker (VVI or VVIR), and 12 a dual chamber pacemaker (DDD). The number of intracardiac leads was as follows: one lead in seven patients, two leads in 14 patients, three leads in one patient, and four leads in one patient. No antimicrobial prophylaxis was given before implantation. At the time of admission to our institution, one patient had an isolated low grade fever and the other 22 had chills, prolonged fever, and positive blood cultures. Infection was secondary to the first implantation in 10 patients. Signs of local inflammation or abscess over the pulse generator pocket were present in only six patients at the time of diagnosis. The portal of entry was unrelated to the pulse generator in three patients and remained unknown in 14 . Septic pulmonary embolism revealed the infection in one patient. Of the 23 patients, two had early $(9 \%)$, four intermediate $(17 \%)$, and 17 late onset infection (74\%). The mean (SD) delay from the last operation to the first signs of infection was 26.2 (32.2) months (range 0 to 120). The interval between the onset of symptoms and the diagnosis was 2.6 (3.8) months (range three days to 15 months).

Table 2 Microbiological data and visualisation of vegetation on echocardiography

\begin{tabular}{|c|c|c|c|c|c|}
\hline Case & Causative bacteria & Lead culture & $\begin{array}{l}\text { Vegetation(s) } \\
\text { TOE / TTE }\end{array}$ & Vegetation type & $\begin{array}{l}\text { Valvar } \\
\text { endocarditis }\end{array}$ \\
\hline 1 & Staphylococcus epidermidis & + & $+1+$ & 3 & - \\
\hline 2 & Staphylococcus epidermidis & + & $+1-$ & 3 & - \\
\hline 3 & Staphylococcus epidermidis & Not done & $+1-$ & 3 & - \\
\hline 4 & Staphylococcus hominis & - & $+1+$ & 2 & - \\
\hline 5 & Staphylococcus epidermidis & + & $+1+$ & 1 & - \\
\hline 6 & Staphylococcus epidermidis and $S$ aureus & + & $+1-$ & 3 & - \\
\hline 7 & Staphylococcus aureus & + & $+1-$ & 1 & - \\
\hline 8 & Streptococcus bovis & + & $+1-$ & 2 & Mitral \\
\hline 9 & Staphylococcus epidermidis & Not done & $+1-$ & 2 & - \\
\hline 10 & Staphylococcus epidermidis & + & $+1-$ & 3 & - \\
\hline 11 & Staphylococcus epidermidis & + & $+1+$ & 3 & \\
\hline 12 & Staphylococcus epidermidis & + & $+1-$ & 3 & - \\
\hline 13 & Staphylococcus capitis & - & $+1+$ & 1 & - \\
\hline 14 & Serratia marcescens & $+^{\star}$ & $+1-$ & 2 & - \\
\hline 15 & Staphylococcus epidermidis & + & $+1-$ & 2 & - \\
\hline 16 & Staphylococcus aureus & + & $+1+$ & 2 & - \\
\hline 17 & Candida glabrata & + & $+\star \star /-$ & $\star \star$ & Trisupid, aortic \\
\hline 18 & Staphylococcus epidermidis & + & $+1-$ & 1 & - \\
\hline 19 & Staphylococcus epidermidis & + & $+1-$ & 2 & - \\
\hline 20 & Staphylococcus epidermidis & + & $+1-$ & 2 & - \\
\hline 21 & Staphylococcus aureus & + & $-1-$ & - & - \\
\hline 22 & Streptococcus agalactiae & + & $+1+$ & 3 & - \\
\hline 23 & Streptococcus bovis & - & $+1-$ & 3 & - \\
\hline
\end{tabular}

* Positive only for the proximal tip of the lead; ${ }^{\star \star}$ on a tricuspid leaflet, not on the lead; vegetation type: see text. 


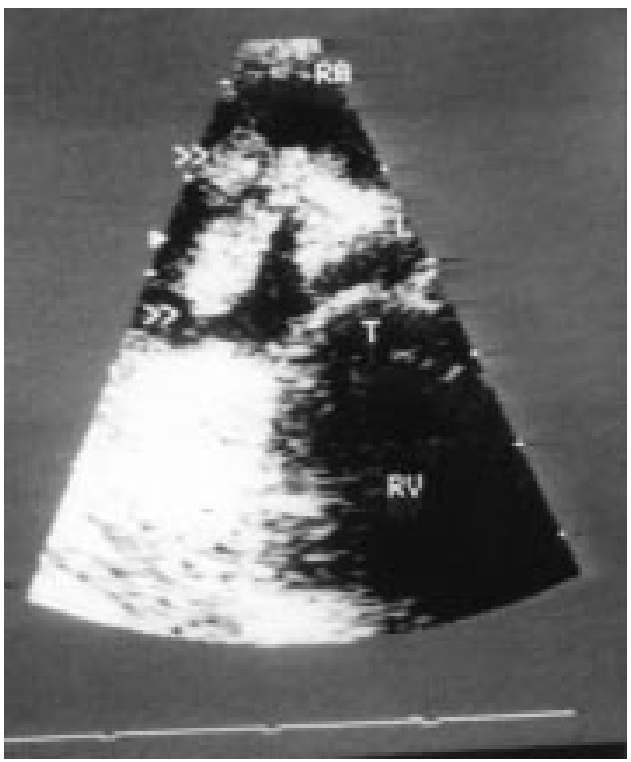

Figure 1 Transoesophageal echocardiography showing a type 1 vegetation close to an intact tricuspid valve. Note the flattening of the atrial lead. L, lead; $R A$, right atrium; $R V$, right ventricle; $T$, tricuspid valve; $>>$ indicates the vegetation.

\section{BACTERIOLOGICAL DATA}

Bacteriological data are given in table 2. Blood cultures were negative in one patient. Twenty three microorganisms were isolated from blood cultures of the other 22 patients (a single blood culture was positive in one patient). The responsible microorganism was mostly a staphylococcus species: $S$ epidermidis in 12 patients, coagulase negative staphylococcus in two, $S$ aureus in three, and mixed $S$ aureus and $S$ epidermidis in one. Other cases included Streptococcus bovis $(\mathrm{n}=2)$, Streptococcus agalactiae $(\mathrm{n}=1)$, Candida glabrata $(\mathrm{n}=1)$, and Serratia marcescens $(\mathrm{n}=1)$. All the patients had explantation of the entire pacing system but culture of the leads was not performed in two cases. The causative microorganism was recovered from the leads in 17 of the 20 patients who had positive blood cultures and evaluable lead culture $(85 \%)$. In addition, of the 20 patients

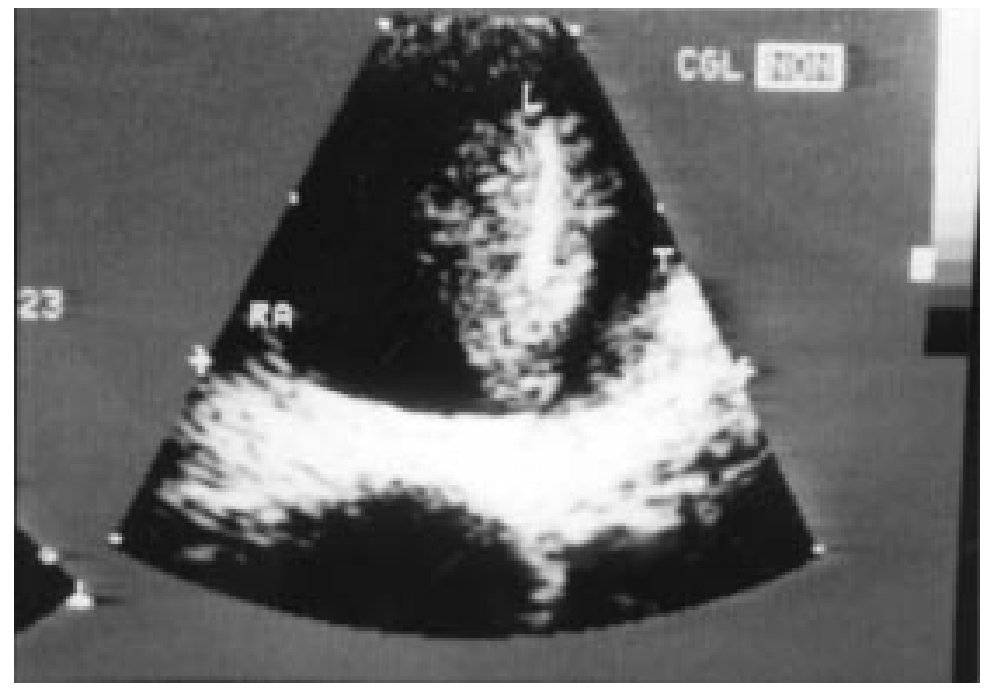

Figure 2 Transoesophageal echocardiography showing a type 2 vegetation in the right atrium surrounding the atrial lead and adjacent to the tricuspid. $L$, lead; $R A$, right atrium; T, tricuspid valve. with TOE detected vegetation in whom lead culture was available, 17 had a positive lead culture (85\%) (table 2).

ECHOCARDIOGRAPHIC DATA

TTE identified right atrial neostructures among the multiple lead induced echoes in seven patients $(30 \%)$. It was, however, difficult to distinguish precisely between abnormal masses, the tricuspid valve, and the lead (table 2).

In contrast, TOE explored the entire intracardiac route of the leads, from the upper vena cava lumen to the right ventricular apex. One patient with bacteriologically confirmed early onset lead infection had no vegetations (case 21). Vegetations were visualised in the 22 remaining cases. In addition, TOE made it possible to locate the vegetations on the lead precisely - on the atrial part of the leads in 10 patients, in the atrioventricular position in seven, on the right ventricular portion of the lead in three, and at the coronary sinus ostium in one. Except in two cases, the vegetative lesions were strictly localised on the lead, even when the tricuspid valve and the neostructure were in close contact. One patient had mitral valve endocarditis complicated by secondary localisation on the pacemaker lead documented by two successive TOE examinations. The other patient had both aortic and tricuspid valve endocarditis. In this patient, the vegetation seemed to be attached to a tricuspid leaflet and not to the lead (table 2).

Three types of lead vegetations were identified:

Type 1-Multiple vegetations with fine pedicles, variably lobular $(\mathrm{n}=4)$ (fig 1$)$.

Type 2-A single, round shaped pedicular growth adhering to the lead $(n=8)$ (fig 2$)$.

Type 3-Lone or multiple thick floating strip(s) fixed to the lead ( $n=9$ ) (fig 3 ).

The size of the vegetations, especially types 1 and 3, was not easily quantified because of their morphological complexity. Nonetheless the mean orthogonal axes were measured to $16 / 30 \mathrm{~mm}$ (range 5 to $25 / 15$ to $30 \mathrm{~mm}$ ) for type $1,12 / 16 \mathrm{~mm}$ (range 7 to $20 / 8$ to $30 \mathrm{~mm}$ ) for type 2 , and $11 / 20 \mathrm{~mm}$ (range 6 to $20 / 15$ to 30 $\mathrm{mm}$ ) for type 3 vegetations. These vegetations of variable echogenicity were attached to an echodense and thickened lead. They were highly mobile and sometimes prolapsed through the tricuspid valve. For vegetation analysis, each operator correctly classified them as type 1,2 , or 3 . Because of the morphological complexity of the neostructures, the variation in measurements averaged $10 \%$.

In the control group, strands were observed in five patients $(29 \%)$. Four patients had a single strand and one had two echodense strands, 1-2 mm wide and 3-5 mm long, attached to the leads. All were found along the intra-atrial lead route, none in the ventricle. The triscupid valve was normal in this group.

In this series, the sensitivity of TTE and TOE was $30 \%$ and $96 \%$ respectively. The specificity and positive predictive value of TOE were both $100 \%$. The negative predictive value of TOE was $94 \%$. 


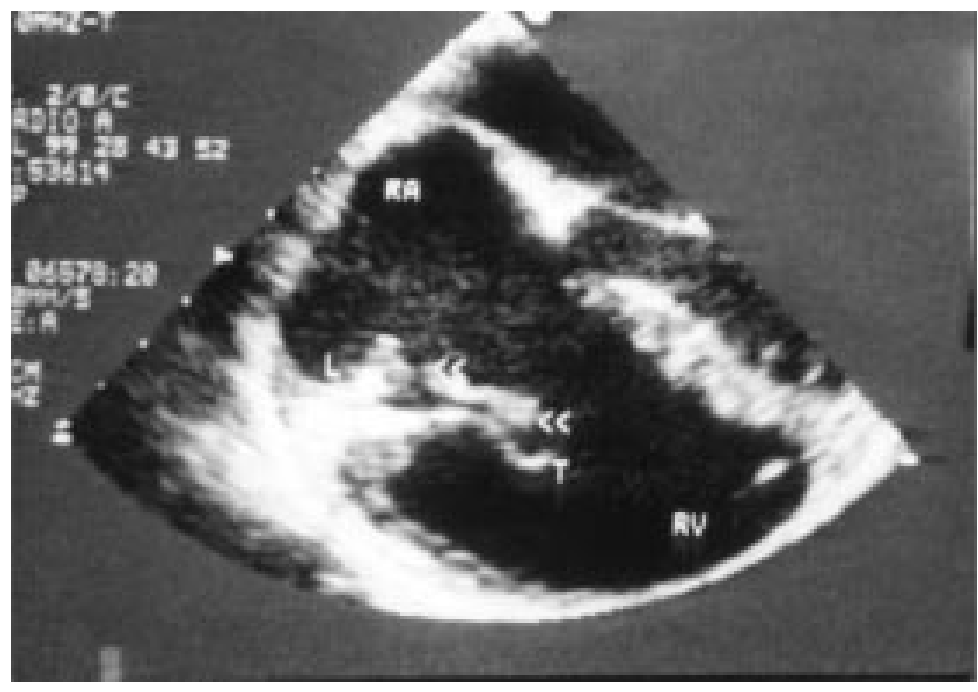

Figure 3 Transoesophageal echocardiography showing a type 3 vegetation in the right atrium close to the tricuspid valve but without valvar endocarditis. $L$, lead; $R A$, right atrium; $R V$, right ventricle; $T$, tricuspid valve; $>>$ indicates the vegetation.

Table 3 Treatment, follow up, and outcome of the 23 patients

\begin{tabular}{|c|c|c|c|}
\hline Case & $\begin{array}{l}\text { Explantation } \\
\text { procedure }\end{array}$ & Reimplantation & $\begin{array}{l}\text { Follow up (months) } \\
\text { and outcome }\end{array}$ \\
\hline 1 & $\mathrm{M} / \mathrm{S}$ & No & 55, survived \\
\hline 2 & $M$ & Epicardial & 70 , survived \\
\hline 3 & M & Epicardial & 48 , survived \\
\hline 4 & $M$ & Patient refused & Lost to follow up \\
\hline 5 & M & Epicardial & 44 , survived \\
\hline 6 & $\mathrm{M} / \mathrm{S}$ & Epicardial & 48 , survived \\
\hline 7 & M & Epicardial & 42 , survived \\
\hline 8 & $\mathrm{~S}$ & Epicardial & 39 , survived \\
\hline 9 & M & Endovascular & 44 , survived \\
\hline 10 & $\mathrm{M} / \mathrm{S}$ & Epicardial & 38 , survived \\
\hline 11 & $\mathrm{M}$ & Endovascular & 9, died \\
\hline 12 & $\mathrm{~S}$ & No & 30 , survived \\
\hline 13 & $\mathrm{~S}$ & No & 24 , survived \\
\hline 14 & $\mathrm{~S}$ & Epicardial & 24 , died \\
\hline 15 & M & Endovascular & 21 , survived \\
\hline 16 & $\mathrm{M}$ & Epicardial & 12 , survived \\
\hline 17 & $M$ & No & 2 , died \\
\hline 18 & $\mathrm{M} / \mathrm{S}$ & Epicardial & 9 , survived \\
\hline 19 & $M$ & No & 9 , survived \\
\hline 20 & $M / S$ & No & 10 , survived \\
\hline 21 & $\mathrm{M}$ & No & 14 , survived \\
\hline 22 & M & Epicardial & 20, died \\
\hline 23 & $\mathrm{M}$ & No & 4 , survived \\
\hline
\end{tabular}

M, medical endovascular extraction; S, surgical extraction.

TREATMENT AND OUTCOME

Results of treatment are shown in table 3. In four patients, surgery was attempted as the procedure of first choice because of the need to perform valve replacement (one case), because of intracavitary lead migration (two cases), and because of previous clinical pulmonary embolism confirmed by scintigraphy (one case). Medical extraction was performed in the other 19 patients using stylets and sheets if necessary. Fourteen procedures (73.7\%) were successful in extracting the entire infected pacing system, but in only one patient could a type 3 vegetation be recovered from the extracted lead. In this particular case, we did not use sheets, which probably sheared off the vegetations attached to the leads in the other cases. The five procedures that failed (where extraction was incomplete or impossible) led to thoracic surgery. In 12 patients with vegetations of $\geqslant 10 \mathrm{~mm}$, medical extraction was attempted and was successful in nine. None of the patients had clinical signs of pulmonary embolism complicating the medical extraction.

This plan of treatment resulted in cure of infection in 22 patients (96\%). One patient was considered cured on discharge from hospital but was lost of follow up. Only one patient died-of uncontrolled Candida glabrata endocarditis two months after pacemaker removal. Three patients died of heart failure but without recurrent infection at 9,20 , and 24 months after explantation. All the other patients remained free from relapse after a follow up of 31.2 (19.1) months (range 4 to 70 months) (table 3).

\section{Discussion}

In this series, using TOE we visualised three types of vegetations on the lead in 21 patients $(91 \%)$ and a vegetation on the tricuspid valve in another patient, while TTE gave positive results in only seven patients $(30 \%)$. Culture of the explanted leads led to isolation of the microorganism recovered from the blood cultures in 17 of 20 cases (85\%). Associated left sided valvar endocarditis was diagnosed in two cases $(9 \%)$. In the control series, only five of the 17 patients $(29 \%)$ showed strands along the atrial route of the lead. No valvar abnormality was observed in this group. Medical extraction of large lead vegetations appeared to be possible and safe.

ADVANTAGES AND LIMITATIONS OF TTE

In a review of published reports, Kugener et al reported the results of TTE obtained in 42 cases of permanent endocavitary lead infection. ${ }^{9}$ The sensitivity of the technique was poor, and it provided a positive diagnosis in only 15 of the cases reviewed (36\%). Such a low sensitivity can be explained by the use of the TM mode in the earliest cases. With the two dimensional mode, the main technical difficulties - apart from the poor echogenicity in some patients - were the echoes induced by the leads which interfere with the visualisation of small sized structures. In addition, when vegetations were visualised, it was generally not possible to determine whether there was triscupid valve endocarditis, lead infection, or both. According to Klug et al, diagnosis of vegetations was possible with TTE in only $23 \%$ of the cases. ${ }^{5}$ Our results were similar, since we visualised abnormal structures in contact with the leads in only seven cases (30\%) using TTE.

ADVANTAGES AND LIMITATIONS OF TOE

Cases of lead infection identified by TOE have recently been published..$^{56-13}$ Few studies have compared TOE with TTE. ${ }^{5}{ }^{12} 13$ According to Klug et al, only $24 \%$ of the vegetations found by TOE were seen by TTE $(11 / 46) .{ }^{5}$ In that series, TOE showed vegetations in $84 \%$ of the patients. In our present series, TOE showed lead vegetations in 21 patients $(91 \%)$. Since we only investigated patients classified as having definite pacemaker lead infection, there may have been an overestimation of TOE sensitivity, because a positive TOE was required for the diagnosis in most cases. TOE permits accurate study of the other valves and the 
situation, insertion, and size of vegetations. The use of a multiplane echographic probe undoubtedly improves the quality of exploration. Indeed, pacing leads shift from their position at each cardiac cycle and in different planes, and these shifts cannot be analysed simultaneously with single or biplane methods. However, because we did not use both types of probe in the same patient, we cannot conclude definitively that multiplane probes are superior. The two patients in whom we were unable to detect any neostructure on the leads with TOE were those in whom the bloodstream infection immediately followed primary implantation. It is therefore possible that the delay to the diagnosis was shorter than required for the growth of detectable vegetations. In cases of negative TOE examination during the early days of possible infection, with no inflammation over the pulse generator pocket, a second exploration may be necessary. However, an extracardiac localisation of the infected focus on the endovascular route may also account for negative results of TOE.

VEGETATION MORPHOLOGY AND SITUATION

In the series of Vilacosta $e t a l,{ }^{12}$ the vegetation size (mean (SD)) was 18 (5) $\mathrm{mm}$ (single plane TOE) and tricuspid endocarditis was reported in only one case. In our series the size of the vegetations depended on their type but was in the same range. For the leads that were surgically removed, and in one example after medical extraction, the morphological appearance of the vegetations was similar to the image obtained with TOE. In contrast, relatively small sized vegetations were common in Klug et al's series: $\geqslant 5$ but $<10 \mathrm{~mm}$ in $51 \%$ of the patients and $<5 \mathrm{~mm}$ in $12 \%$ of the patients in whom a vegetation was observed..$^{5}$ Tricuspid valve vegetation was documented in only one of our patients (case 17). Surprisingly, in this case TOE did not reveal lead vegetation. Nonetheless, tricuspid valve endocarditis may exist even when TOE does not show valve vegetations. We also observed mitral valve endocarditis (case 8) and aortic valve endocarditis (case 17). In the first patient, there was no evidence of tricuspid valve involvement in the TOE examination.

SPECIFICITY OF TOE IMAGES

In Klug et al's series, $13 \%$ of patients (seven of 52) did not have definite infective endocarditis on the basis of the Duke criteria, and the lead culture was negative in $22 \%$ (11 of 49 ). ${ }^{5}$ The investigators explain these observations by the role of preoperative antibiotic treatment. In the present series, the same microorganism was isolated in lead culture as in blood cultures in 17 of the 20 cases $(85 \%)$ and the lead culture was positive in $86 \%$ of all cases. This confirmed the infectious nature of the neostructures visualised. Such vegetative images were not observed in the control group, though thin strands could be seen adhering to intracardiac leads in the right intra-atrial position. Whereas strands are commonly observed by TOE on prosthetic heart valves, especially mitral valves, ${ }^{8-14}$ their significance remains unknown in patients with endovascular leads. In addi- tion, these strands are not always detected during successive examinations of the same patient, which makes their evaluation all the more difficult. ${ }^{8}$ Lastly, although they are associated with a risk of peripheral embolism, their exact nature (fibrin, thrombus, pannus, etc) is still unknown. Whether pacemaker lead associated strands promote the development of infection remains hypothetical.

In a retrospective study involving 39 patients, Lauck et al reported the presence of structures defined as "sessile" and "thrombotic" attached to pacemaker leads. ${ }^{15}$ They were only observed by TOE in $31 \%$ of cases. These investigators identified atrial fibrillation, a left ventricular ejection fraction below $40 \%$, and spontaneous echographic contrast as factors associated with the presence of such structures. However, the presence or absence of signs of sepsis was not specified, and it was not possible to determine whether these neostructures were bacterial vegetations or whether they were thrombotic.

\section{CLINICAL IMPLICATIONS}

Ten years ago, the mortality from pacemaker infection in the absence of explantation was estimated to be $66 \% .^{1}$ This emphasises the importance of rapid and accurate diagnosis leading to the removal of the entire pacing system as soon as possible. ${ }^{4}$ The clinical presentation is often atypical, with isolated prolonged fever. Blood cultures are usually positive, but the source of infection is frequently unknown. In the present series, all patients were classified as definite infective endocarditis according to the Duke criteria. ${ }^{16}$ All but one had two major criteria, with positive blood cultures and a positive echocardiogram for infective endocarditis. The patient without positive blood cultures had a positive echocardiogram and a positive lead culture and could also be classified as definite infective endocarditis. In contrast, in the control series no patient had a positive echocardiogram for infective endocarditis, suggesting that strands attached to the pacemaker leads can exist "physiologically". Finally, whenever a pacemaker lead infection is suspected, TOE is indicated because of the poor sensitivity of TTE and because this examination makes a major contribution to the diagnosis and the precise characterisation of the lesions. In addition, few other imaging techniques are available. Magnetic resonance imaging is contraindicated in pacemaker carriers. Computed tomography has not been evaluated. Positive diagnosis with indium labelled leucocyte scanning is anecdotal.

In Klug et al's study, the shape and size of the vegetations did not influence the clinical outcome, and there was no significant relation between pulmonary migration and vegetation size. ${ }^{5}$ Medical extraction of vegetations above $10 \mathrm{~mm}$ was attempted in five patients in that series, with scintigraphic evidence of pulmonary embolism in two cases $(40 \%)$, but only one patient (with vegetations of $40 \mathrm{~mm}$ ) was symptomatic. All five patients survived. In our series, the success of medical extraction in case of vegetations $\geqslant 10 \mathrm{~mm}$ was $75 \%$. Subclinical 
embolism was not ruled out by systematic scintigraphy, but the outcome after lead removal was unremarkable in all these patients.

CONCLUSION

Pacemaker lead infection is a serious complication of cardiac pacing. The absence of local signs of infection over the pulse generator pocket is common, making the diagnosis all the more difficult. Normal TTE images do not rule out lead infection because of the low sensitivity of the technique. TOE, which identifies three types of vegetation, appears to be a determining procedure. Combined with bacteriological results, TOE images strongly suggest pacemaker lead infection and should lead to total explantation of the pacing system. Although we could not assess the risk of embolism in relation to the size and type of the vegetations, medical extraction was often successful and proved safe in our series.

1 Phibbs B, Marriot HJL. Complications of permanent transvenous pacing. N Engl f Med 1985;312:1428-32.

2 Conklin EF, Giannelli S, Nealon TF. Four hundred consecutive patients with permanent transvenous pacemakers. F Thorac Cardiovasc Surg 1975;69:1-7.

3 Bluhm G. Pacemaker infections: a clinical study with special reference to prophylactic use of some isoxazolyl penicillins. Acta Med Scand Suppl 1985;699:1-62.

4 Camus C, Leport C, Raffi F, et al. Sustained bacteremia in 26 patients with a permanent endocardial pacemaker: assessment of wire removal. Clin Infect Dis 1993;17:46-55.
5 Klug D, Lacroix D, Savoye C, et al. Systemic infection Klug D, Lacroix D, Savoye C, et al. Systemic infection related to endocarditis on pacemaker leads. Clinical
presentation and management. Circulation 1997;95:2098107.

6 Vilacosta I, Zamorano J, Camino A, et al. Infected transvenous permanent pacemakers: role of transesophageal echocardiography. Am Heart f 1993;125:904-6.

7 Lewis AB, Hayes DL, Holmes DR, et al. Update on infections involving permanent pacemaker infections: characterization and management. 7 Thorac Cardiovasc Surg 1985;89:758-63.

8 Orsinelli DA, Pearson AC. Detection of prosthetic valve strands by transesophageal echocardiography: clinical significance in patients with suspected cardiac source of embolism. F Am Coll Cardiol 1995;26:1713-18.

9 Kugener H, Rey JL, Tribouilloy C, et al. Endocardites infectieuses sur sonde de stimulateur endocavitaire permanente: intérêt de l'échographie transoesophagienne. Ann Cardiol Angeiol 1993;42:331-8.

10 Zehender M, Buchner C, Geibel, et al. Diagnosis of hidden pacemaker lead sepsis by transesophageal echocardiography and a new technique for lead extraction. Am Heart f 1989;118:1050-3.

11 Van Camp G, Vandenbossche JL. Recognition of pacemaker lead infection by transesophageal echocardiography. $\mathrm{Br}$ Heart f 1991;65:229-30.

12 Vilacosta I, Sarria C, San Roman JA, et al. Usefulness of transesophageal echocardiography for diagnosis of infected transvenous permanent pacemaker. Circulation 1994;89: 2684-7.

13 Victor F, De Place C, Leclercq C, et al. Infections sur sonde de stimulation endocavitaire permanente: intérêt de l'échographie transoesophagienne. Arch Mal Coeur 1995; 88:1875-81.

14 Freedberg RS, Goodkin GM, Perez JL, et al. Valve strands are strongly associated with systemic embolization: a trans-
esophageal echocardiographic study. $\mathcal{f}$ Am Coll Cardiol 1995;26:1709-12.

15 Lauck G, Hagendorff A, Omran H, et al. Sessile structures on implanted leads of cardiac pacemakers [abstract]. Eur Heart $\mathcal{F}$ 1994;15:58.

16 Bayer A, Ward J, Ginzton L, et al. Evaluation of new clinical criteria for the diagnosis of infective endocarditis. Am $\mathcal{F}$ Med 1994;96:211-19. 